\title{
Prognostic significance of FDG-Positron Emission Tomography (FDG-PET) response post chemotherapy and definitive radiotherapy in pediatric rhabdomyosarcoma
}

\author{
BADIRA CHERIYALINKAL PARAMBIL ${ }^{1}$, Sneha Shah ${ }^{1}$, Tushar Vora ${ }^{1}$, Maya Prasad ${ }^{1}$, \\ Siddhartha Laskar ${ }^{1}$, Nehal Khanna ${ }^{1}$, Sajid Qureshi ${ }^{1}$, Mukta Ramadwar ${ }^{1}$, Seema \\ Kembhavi $^{1}$, Hari Sankaran ${ }^{1}$, Venkatesh Rangarajan ${ }^{1}$, and Girish Chinnaswamy ${ }^{1}$ \\ ${ }^{1}$ Tata Memorial Hospital
}

September 28, 2020

\begin{abstract}
1 Background Persisting residual mass at treatment completion are known in rhabdomyosarcoma(RMS) who have been treated with definitive radiotherapy to the primary site, but their prognostic significance is uncertain. Tumour response as assessed by anatomic imaging is not prognostic and there are only limited studies based on FDG-PET response. We report the prognostic significance of persistent FDG avidity in residual masses, assessed 3-months post completion of radiotherapy, in paediatric RMS who have undergone definitive RT as primary local therapy. 2 Materials and Methods Children[?]15 years with Group 3 or 4 RMS treated on a uniform chemotherapy protocol, who received definitive radiotherapy for local control from June 2013December 2018, and had FDG-PET CT at 3-months post radiotherapy were retrospectively analysed for outcomes. 3 Results Sixty-three children formed the study cohort, (55 Group3 and 8 Group4) FDG-PET CT scan done 3-months post-radiotherapy showed FDG-avid residual mass in 11 patients(17.5\%), morphologic only (FDG negative) residual mass in 24 patients(38.1\%) and no residual in 28 patients(44.4\%). At a median follow-up of 41months (range,10-83months), 3-year Event Free Survival of patients with FDG-avid residual are $45.5 \%$ (95\%CI:23.8\%-86.8\%) and for those with morphologic only or no residual are $71.4 \%$ (95\%CI:59.6\%-85.5\%). Presence of FDG-avid residual on PET-CT scan 3-months post definitive RT [HR-2.92(95\%CI:1.13$7.57), \mathrm{p}=0.028$ ] and regional lymph node involvement [HR-3.14(95\%CI:1.26-7.78), $\mathrm{p}=0.014$ ] affected outcomes, which retained significance on multivariate analysis too. 4 Conclusions Persistent metabolic activity in residual disease at the end of therapy in RMS may portend poorer prognosis, and help identify patients who would benefit from alternative treatment strategy.
\end{abstract}

\section{TITLE PAGE}

\section{COMPLETE TITLE:}

Prognostic significance of FDG-Positron Emission Tomography (FDG-PET) response post chemotherapy and definitive radiotherapy in pediatric rhabdomyosarcoma

\section{AUTHOR LIST WITH AFFILIATIONS:}

Badira Cheriyalinkal Parambil, MD, DM, Department of Pediatric Oncology, Tata Memorial Hospital, Homi Bhabha National Institute (HBNI), Mumbai, India

Sneha Shah, MD, Department of Nuclear Medicine, Tata Memorial Hospital, Homi Bhabha National Institute (HBNI), Mumbai, India

Tushar Vora, MD, Department of Pediatric Oncology, Tata Memorial Hospital, Homi Bhabha National Institute (HBNI), Mumbai, India 
Maya Prasad, MD, Department of Pediatric Oncology, Tata Memorial Hospital, Homi Bhabha National Institute (HBNI), Mumbai, India

Siddhartha Laskar, MD, Department of Radiation Oncology, Tata Memorial Hospital, Homi Bhabha National Institute (HBNI), Mumbai, India

Nehal Khanna, MD, Department of Radiation Oncology, Tata Memorial Hospital, Homi Bhabha National Institute (HBNI), Mumbai, India

Sajid Qureshi, MS, Department of Surgical Oncology, Tata Memorial Hospital, Homi Bhabha National Institute (HBNI), Mumbai, India

Mukta Ramadwar, MD, Department of Pathology, Tata Memorial Hospital, Homi Bhabha National Institute (HBNI), Mumbai, India

Seema Kembhavi, MD, Department of Radiodiagnosis, Tata Memorial Hospital, Homi Bhabha National Institute (HBNI), Mumbai, India

Hari Sankaran, MD, Department of Pediatric Oncology, Tata Memorial Hospital, Homi Bhabha National Institute (HBNI), Mumbai, India

Venkatesh Rangarajan, MD, Department of Nuclear Medicine, Tata Memorial Hospital, Homi Bhabha National Institute (HBNI), Mumbai, India

Girish Chinnaswamy, MD, Department of Pediatric Oncology, Tata Memorial Hospital, Homi Bhabha National Institute (HBNI), Mumbai, India

\section{CORRESPONDING AUTHOR:}

Name: Prof. Girish Chinnaswamy

Address: Department of Pediatric Oncology, Main Building, Tata Memorial Hospital, Mumbai, India, 400012

Phone: $+22-24177000$

Email: girish.c.tmh@gmail.com

\section{WORD COUNT:}

Abstract: 250

Main Text: 2213

Tables: 3, Figures: 3

\section{SHORT RUNNING TITLE:}

Prognostic significance of FDG-PET post definitive radiotherapy in rhabdomyosarcoma

KEYWORDS:

FDG-PET, Rhabdomyosarcoma, FDG-avid residual, definitive radiotherapy

\section{ABBREVIATIONS:}

FDG-PET Fluorodeoxyglucose-Positron Emission Tomography

RMS Rhabdomyosarcoma

EFS Event-Free Survival

OS Overall Survival

RT Radiotherapy 
IMRT Intensity modulated radiotherapy

\section{DATA AVAILABILITY STATEMENT:}

The data that support the findings of this study are available from the corresponding author upon reasonable request.

Prognostic significance of FDG-Positron Emission Tomography (FDG-PET) response post chemotherapy and definitive radiotherapy in pediatric rhabdomyosarcoma

Badira Cheriyalinkal Parambil ${ }^{1}$, Sneha Shah ${ }^{2}$, Tushar Vora ${ }^{1}$, Maya Prasad ${ }^{1}$, Siddhartha Laskar ${ }^{3}$, Nehal Khanna $^{3}$, Sajid Qureshi, ${ }^{4}$ Mukta Ramadwar ${ }^{5}$, Seema Kembhavi ${ }^{6}$, Hari Sankaran ${ }^{1}$, Venkatesh Rangarajan ${ }^{2}$, Girish Chinnaswamy ${ }^{1}$

${ }^{1}$ Department of Pediatric Oncology, Tata Memorial Hospital, Homi Bhabha National Institute (HBNI), Mumbai

${ }^{2}$ Department of Nuclear Medicine, Tata Memorial Hospital, Homi Bhabha National Institute (HBNI), Mumbai

${ }^{3}$ Department of Radiation Oncology, Tata Memorial Hospital, Homi Bhabha National Institute (HBNI), Mumbai

${ }^{4}$ Department of Surgical Oncology, Tata Memorial Hospital, Homi Bhabha National Institute (HBNI), Mumbai

${ }^{5}$ Department of Pathology, Tata Memorial Hospital, Homi Bhabha National Institute (HBNI), Mumbai

${ }^{6}$ Department of Radiodiagnosis, Tata Memorial Hospital, Homi Bhabha National Institute (HBNI), Mumbai

\section{ABSTRACT}

\section{Background}

Persisting residual mass at treatment completion are known in rhabdomyosarcoma(RMS) who have been treated with definitive radiotherapy to the primary site, but their prognostic significance is uncertain. Tumour response as assessed by anatomic imaging is not prognostic and there are only limited studies based on FDG-PET response. We report the prognostic significance of persistent FDG avidity in residual masses, assessed 3-months post completion of radiotherapy, in paediatric RMS who have undergone definitive RT as primary local therapy.

\section{Materials and Methods}

Children[?]15 years with Group 3 or 4 RMS treated on a uniform chemotherapy protocol, who received definitive radiotherapy for local control from June 2013-December 2018, and had FDG-PET CT at 3-months post radiotherapy were retrospectively analysed for outcomes.

\section{Results}

Sixty-three children formed the study cohort, (55 Group3 and 8 Group4) FDG-PET CT scan done 3months post-radiotherapy showed FDG-avid residual mass in 11 patients(17.5\%), morphologic only (FDG negative) residual mass in 24 patients(38.1\%) and no residual in 28 patients(44.4\%). At a median followup of 41months (range,10-83months), 3-year Event Free Survival of patients with FDG-avid residual was 45.5\% (95\%CI:23.8\%-86.8\%) and for those with morphologic only or no residual was $71.4 \%$ (95\%CI:59.6\%85.5\%). Presence of FDG-avid residual on PET-CT scan 3-months post definitive RT [HR-2.92(95\%CI:1.13$7.57), \mathrm{p}=0.028$ ] and regional lymph node involvement [HR-3.14(95\%CI:1.26-7.78), $\mathrm{p}=0.014]$ affected outcomes, which retained significance on multivariate analysis too.

\section{Conclusions}


Persistent metabolic activity in residual disease at the end of therapy in RMS may portend poorer prognosis, and help identify patients who would benefit from alternative treatment strategy.

\section{INTRODUCTION}

Rhabdomyosarcoma (RMS) accounts for $3.5 \%$ and $2 \%$ of malignancies in 0-14 years and 15-19 years age groups respectively with well-established prognostic factors which include age, size, site, histology, molecular status, clinical group and stage.(1-12) Most clinical group III patients undergo definitive radiotherapy (RT) for local control, and persistence of mass at the completion of all therapy is well known. Initial retrospective studies carried out on the biopsy specimens of subsets of patients with RMS and residual tumor masses showed the presence of mature rhabdomyoblasts and did not justify radical surgeries or continuation of therapy.(13-14) These radiographic masses can result from stromal scarring or cytodifferentiation of RMS cells to rhabdomyoblasts post chemo-radiotherapy and has been shown to be of no prognostic significance when assessed by anatomic imaging in a larger IRS-IV (Intergroup Rhabdomyosarcoma) study cohort.(15) Functional imaging using ${ }^{18} \mathrm{~F}$-FDG-PET (fluorodeoxyglucose positron emission tomography) has been used in response assessment in limited settings such as Hodgkin lymphoma in pediatric oncology to tailor treatment, especially due to their ability to better differentiate viable versus necrotic tissue.(16) Such studies utilizing PET for prognostication at treatment completion are limited in RMS and are heterogeneous with respect to the time point of assessment.(17-18) In this study, we retrospectively analysed the prognostic significance of persistent FDG avidity in residual masses in paediatric patients with RMS. All patients had undergone definitive RT as primary local therapy, and were analysed at a specific time point post-RT.

\section{AIMS AND OBJECTIVES}

Patients treated for RMS at the pediatric oncology department of our center over a 5.5-year period (June 2013-December 2018) using a uniform protocol and who received radiation therapy as definitive local treatment were included. The ${ }^{18} \mathrm{~F}$-FDG-PET CT scans performed at 3-month post completion of radiotherapy were analyzed retrospectively. The primary objective was to assess the prognostic significance of residual masses for event free survival (EFS). Secondary objectives were to study the overall survival (OS), incidence of residual masses and clinical profile of the cohort.

\section{MATERIALS AND METHODS}

\subsection{Methods}

Children ([?]15 years) with biopsy proven, clinical group 3 or 4 RMS from June 2013 to December 2018 and who received radiation therapy as definitive local treatment were included. Those who could not complete treatment due to progression or toxic death were excluded. The study cohort had FDG-PET CT scan done 3months post radiotherapy. Diagnosis was made by histopathology (including immunohistochemistry). Molecular translocation studies (fusion status studies) for PAX3-FKHFR and PAX7-FKHFR was done whenever possible. Staging was done by PET-CT scan and bilateral bone marrow aspiration and biopsies in all.

Lymph node sampling and CSF studies for staging (in para-meningeal primary) and MRI (Magnetic Resonance Imaging) for better delineation of primary tumor were performed where indicated. All patients were treated on a uniform chemotherapy protocol consisting of 12 cycles of chemotherapy given at 3-weekly intervals [either VIE-vincristine, ifosfamide, etoposide + VCD-vincristine, cyclophosphamide, dactinomycin or VCD only (especially in infants, bladder and prostate RMS or those children with hydroureteronephrosis), Figure 1]. Local therapy was planned after 12 weeks of induction chemotherapy. The eligible cohort received definitive radiotherapy at a dose of 50.4Gy/28\#, 1.8Gy/\# using Intensity modulated RT technique (IMRT). Metastatic sites were addressed with RT at a dose ranging from $41.4 \mathrm{~Gy} / 23 \#$ to $50.4 \mathrm{~Gy} / 28 \#$, dose and fractionation depending on the number of sites and response, usually timed together with RT to primary. Disease status was assessed with ${ }^{18}$ F-FDG-PET CT done 3 months after completion of RT and patients were classified as having either no residual, morphological residual disease with no FDG avidity (defined as no FDG uptake greater than the background blood pool activity) or FDG-avid residual (or metabolic residual, 
defined as FDG uptake greater than the background blood pool activity in a residual lesion).

\subsection{Statistical methods}

Baseline variables and outcomes were analyzed by descriptive statistics. For survival analysis, an event was defined as relapse, progression or death due to any cause. Event-free survival (EFS) was calculated as time from the date of diagnosis to event. Overall survival (OS) was calculated as time from date of diagnosis to death due to any cause, or last follow-up. All patients without an event were censored at last follow up. Estimates of survival were computed using the Kaplan-Meier method. Prognostic variables were analyzed using Cox regression analysis. $\mathrm{P}<0.05$ was considered significant. Statistical analysis was performed using SPSS software, version 24.

\section{RESULTS}

\subsection{Epidemiological and clinical profile}

There were 93 patients who received definitive radiotherapy for local control during the study period. Sixtythree children with a PET-CT assessment 3months post definitive RT, formed the study cohort with a median age of 5 years (range1-15 years) and male to female ratio of 2.1:1. Fifty-five patients had clinical group 3 disease and 8 patients had clinical group 4 disease. The consort diagram of the retrospective study is depicted in Figure 2. The detailed demographic and clinical characteristics are depicted in TABLE 1.

\subsection{Assessment post-definitive radiotherapy by FDG-PET CT}

Patients assessed with FDG-PET CT scan 3-months post-definitive RT showed FDG-avid residual masses in 11 patients (17.5\%), morphological residual in 24 patients (38.1\%) and no residual in 28 patients (44.4\%). Of the 11 patients with FDG-avid residual, 7 patients had residual metabolic activity at the primary site only, 2 had residual metabolic activity at the regional lymph node only, 1 at both primary and focal metastatic site and another 1 at primary and regional lymph node. Three of 8 patients with Group 4 disease had FDG-avid residual (1 each at primary site, regional lymph node, primary and focal bone marrow metastatic site). Of 11 patients with FDG-avid residual, molecular translocation status was available in 9, with fusion positive RMS in 4 (PAX3-FKHFR-3, PAX7-FKHFR-1) and fusion negative RMS in 5.

\subsection{Outcomes}

At the time of analysis, 41 children are alive, 15 expired and 7 are lost to follow-up (last follow up more than 12 months from the date of analysis and not able to contact via telephone). Of 15 deaths in the whole cohort, 14 died due to disease, 1 died while on salvage chemotherapy for relapse, cause unknown. At a median follow up of 41 months (range 10 - 83 months), 3-year EFS and OS of the whole cohort was $66.9 \%$ (95\%CI:55.9\%-80.1\%) and 73.6\% (95\%CI:62.3\%-86.9\%) respectively. Three-year EFS of patients with no residual was $73.3 \%$ (95\%CI:58.0\%-92.6\%), those with morphological only residual was $70.2 \%$ (95\%CI:53.9\%$91.4 \%),(\mathrm{p}=0.964)$ and those with FDG-avid residual was $45.5 \%$ (95\%CI:23.8\%-86.8\%), ( $\mathrm{p}=0.047)$. Since the patients with no residual and morphological residual had similar 3-year EFS, when compared to those patients with FDG-avid residual, they were grouped together for further analysis. Three-year EFS of patients with FDG-avid residual masses was 45.5\% (95\%CI:23.8\%-86.8\%) and for those with morphological residual or no residual was $71.4 \%(95 \% \mathrm{CI}: 59.6 \%-85.5 \%),(\mathrm{p}=0.028)$ and 3-year OS of patients with FDG-avid residual masses was $56.2 \%$ (95\%CI:31.6\%-100.0\%) and for those with morphological residual or no residual was $76.4 \%$ (95\%CI:64.3\%-90.8\%), ( $\mathrm{p}=0.09)$. The survival curves are depicted in Figure 3.

The cumulative incidence of relapse for those with FDG-avid residual was 54.5\% (95\%CI:13.2\%-76.2\%), for those with morphological only residual and no residual were $29.8 \%$ (95\%CI:8.6\%-26.7\%) and $26.7 \%$ (95\%CI:7.4\%-42.0\%) respectively. There were 6 relapses in 11 patients with FDG-avid residual, out of which 4 had FDG-avid residual in primary site only, 1 each in regional lymph-node, primary and metastatic site. Of these 6 relapses, 3 were metastatic lung relapses (1 each with FDG-avid residual on assessment post primary therapy present in primary site only, regional lymph-node only and primary and focal metastatic bone marrow site), 2 were local only relapses (both had FDG-avid residual in primary site only) and 1 was 
regional relapse (FDG-avid residual in primary site only). The median time to relapse in this cohort with FDG-avid residual was 2.5 months (range 0-9 months) from the completion of therapy. The details of the relapses in the study cohort are shown in TABLE 2. Of 6 with FDG-avid residual who relapsed, 3 were fusion positive (PAX3-FKHFR-2, PAX7-FKHFR-1), 1 was fusion negative and translocation studies were not done in 2 .

\subsection{Prognostic factors}

Presence of FDG-avid residual disease persisting after RT [HR-2.92 (95\%CI:1.13-7.57), ( $\mathrm{p}=0.028)$ ] and regional lymph-node involvement [HR-3.14 (95\% CI:1.26-7.78), $(\mathrm{p}=0.014)$ ] affected outcomes in the study population on univariate analysis. On multivariate analysis, FDG-avid residual tumor post definitive RT [HR-2.73 (95\%CI:1.05-7.10), ( $\mathrm{p}=0.04)$ ] and regional lymph-node involvement [HR-3.02 (95\%CI:1.21-7.50), $(\mathrm{p}=0.018)$ ] retained significance for EFS. Molecular translocation status of the tumours was not available in all and hence the prognostic significance could not be ascertained. The details are in TABLE 3 .

\section{DISCUSSION}

Treatment of rhabdomyosarcoma has been refined over successive co-operative group trials, which have defined the risk-adapted approach incorporating the identified prognostic factors. The presence of persistent radiographic masses at the completion of therapy is well known in patients with clinical group 3 RMS treated with definitive radiotherapy and chemotherapy with a reported incidence of $19 \%$ in the IRS IV study cohort.(15) In our study cohort, the incidence of residual mass was $55.6 \%$, higher than the above study and therefore requires a robust tool for prognostication and further management.

Assessment of disease status at treatment completion using anatomic imaging such as CT or MRI has not been found to be of prognostic significance in a COG study, which showed no significant indication of lower risk of failure [HR-0.77,95\%CI:0.46-1.27, $(\mathrm{p}=0.3)$ ] nor death [HR-0.63, 95\%CI:0.36-1.09, $(\mathrm{p}=0.1)$ ] for patients with no residual versus those with anatomic residual. The study also showed that only half of the residual masses that were resected at treatment completion had residual viable tumor and these late surgical resections added to morbidity without improved outcomes.(15) This lack of prognostic significance by anatomic imaging could be due to the inability of these techniques to differentiate necrotic or fibrotic tissue from viable tumor. Hence functional imaging utilizing FDG-PET would be a better tool to identify patients with residual viable tumor and thereby prognosticate these patients. This has been retrospectively analyzed in limited studies, mainly by MSKCC where in positivity on a PET done over a varying time frame ranging from 3 days to 4.5 months after completion of RT was prognostic of LRFS (Local relapse free survival), PFS (Progression free survival) and OS.(17-18) Based on the above studies, and keeping in with the observation from our study that no residual and morphological only residual had similar outcomes, we combined these groups into one for analysis.

In our study, the eligible patients were uniformly assessed at 3 months post definitive RT which would negate the false positive FDG-avidity that could be seen if done earlier due to the metabolic effect post radiation, giving a true estimate of FDG-avid residual. This is also timed towards the latter part of chemotherapy before treatment completion when alternative treatment strategies could be implemented. This is more so relevant taking into consideration that 6 out of the 11 patients with FDG-avid residual relapsed in a period of less than 12 months, most $(n=5 / 6,83.3 \%)$ within 6 months of treatment completion and hence this specific time point of assessment could guide management decisions which is warranted soon thereafter if relapse is to be prevented. This could entail surgical resection of the residual masses if it can be carried out without morbidity and institution of salvage chemotherapy, as the residual disease will already be resistant to the ongoing chemotherapeutic drugs or administration of maintenance chemotherapy in a metronomic fashion after completion of planned chemotherapy and before frank relapse, when the disease burden is low. The latter option appears more attractive than former in the present context where metronomic maintenance chemotherapy has already been shown to improve survival in localized high risk rhabdomyosarcoma and application of salvage chemotherapy with its attendant toxicities might be an overkill and intolerable in this unnavigated area, especially when delivered immediately following primary intensive chemotherapy.(19) As 
the relapses were more metastatic, local therapy in the form of surgical resection alone would not suffice. This was also shown in the MSKCC study where $67 \%$ (18 of 27) of patients with positive post-RT PET relapsed with a local failure rate of $27 \%$ in the cohort.(18) A larger prospective study could inform the most appropriate approach in this context. This study also shows that there is no need for radical surgeries or biopsies in morphological only residual masses on PET-CT.

The limitations of this study are the small sample size and the retrospective nature of the study. But given the number of patients undergoing definitive radiotherapy in this rare disease and the fact that at the patient level, 6 out of the 11 patients with FDG-avid residual relapsed with presence of FDG-avid residual showing prognostic significance on multivariate analysis, there is a compelling evidence warranting alternative strategies for this population and necessitating larger, multicenter prospective studies addressing the issue. In this study, metastatic disease was not prognostically significant, which could probably be due to the lesser number of Group 4 patients in the cohort and will require validation in a larger cohort.

\section{CONCLUSIONS}

Persistent FDG-avid residual masses at 3-months post radiotherapy in paediatric RMS may portend poor prognosis in those receiving definitive local RT. This subset of high risk patients would benefit from alternative treatment strategies, and need to be identified to improve outcomes.

\section{CONFLICT OF INTERESTS}

The authors declare that there is no conflict of interest.

\section{ACKNOWLEDGEMENTS}

The team would like to acknowledge Ms Smruti Mokal for the help provided in statistical analysis.

\section{REFERENCES}

1. Gurney JG, Severson RK, Davis S, et al.: Incidence of cancer in children in the United States. Sex-, race-, and 1-year age-specific rates by histologic type. Cancer 75 (8): 2186-95, 1995.

2. Ries LA, Kosary CL, Hankey BF, et al., eds.: SEER Cancer Statistics Review, 1973-1996. Bethesda, Md: National Cancer Institute, 1999. Also available online. Last accessed August 20, 2019.

3. Malempati S, Rodeberg DA, Donaldson SS, et al.: Rhabdomyosarcoma in infants younger than 1 year: a report from the Children's Oncology Group. Cancer 117 (15): 3493-501, 2011.

4. Sultan I, Qaddoumi I, Yaser S, et al.: Comparing adult and pediatric rhabdomyosarcoma in the surveillance, epidemiology and end results program, 1973 to 2005: an analysis of 2,600 patients. J Clin Oncol 27 (20): 3391-7, 2009.

5. Bradley JA, Kayton ML, Chi YY, et al.: Treatment Approach and Outcomes in Infants With Localized Rhabdomyosarcoma: A Report From the Soft Tissue Sarcoma Committee of the Children's Oncology Group. Int J Radiat Oncol Biol Phys 103 (1): 19-27, 2019.

6. Bisogno G, Compostella A, Ferrari A, et al.: Rhabdomyosarcoma in adolescents: a report from the AIEOP Soft Tissue Sarcoma Committee. Cancer 118 (3): 821-7, 2012.

7. Ferrari A, Miceli R, Meazza C, et al.: Comparison of the prognostic value of assessing tumor diameter versus tumor volume at diagnosis or in response to initial chemotherapy in rhabdomyosarcoma. J Clin Oncol 28 (8): 1322-8, 2010.

8. Spunt SL, Lobe TE, Pappo AS, et al.: Aggressive surgery is unwarranted for biliary tract rhabdomyosarcoma. J Pediatr Surg 35 (2): 309-16, 2000.

9. Crist W, Gehan EA, Ragab AH, et al.: The Third Intergroup Rhabdomyosarcoma Study. J Clin Oncol 13 (3): 610-30, 1995. 
10. Crist WM, Garnsey L, Beltangady MS, et al.: Prognosis in children with rhabdomyosarcoma: a report of the intergroup rhabdomyosarcoma studies I and II. Intergroup Rhabdomyosarcoma Committee. J Clin Oncol 8 (3): 443-52, 1990.

11. Skapek SX, Anderson J, Barr FG, et al.: PAX-FOXO1 fusion status drives unfavorable outcome for children with rhabdomyosarcoma: a children's oncology group report. Pediatr Blood Cancer 60 (9): 1411-7, 2013.

12. Oberlin O, Rey A, Lyden E, et al.: Prognostic factors in metastatic rhabdomyosarcomas: results of a pooled analysis from United States and European cooperative groups. J Clin Oncol 26 (14): 2384-9, 2008.

13. Arndt CA, Hammond S, Rodeberg D, et al: Significance of persistent mature rhabdomyoblasts in bladder/prostate rhabdomyosarcoma: Results from IRS IV. J Pediatr Hematol Oncol 28:563-567, 2006

14. Ortega JA, Rowland J, Monforte H, et al: Presence of well-differentiated rhabdomyoblasts at the end of therapy for pelvic rhabdomyosarcoma: Implications for the outcome. J Pediatr Hematol Oncol 22:106-111, 2000

15. Rodeberg DA, Stoner JA, Hayes-Jordan A, et al. Prognostic significance of tumor response at the end of therapy in group III rhabdo- myosarcoma: A report from the Children's Oncology Group. J Clin Oncol 2009;27:3705-3711.

16. Hutchings M, Loft A, Hansen M, et al. FDG-PET after two cycles of chemotherapy predicts treatment failure and progression-free survival in Hodgkin lymphoma. Blood 2006; 107:52-59.

17. Dharmarajan KV, Wexler LH, Gavane S, et al. Positron emission to- mography (PET) evaluation after initial chemotherapy and radiation therapy predicts local control in rhabdomyosarcoma. Int J Radiat Oncol Biol Phys 2012;84:996-1002.

18. Casey DL, Wexler LH, Fox JJ, Dharmarajan KV, Schoder H, Price AN, Wolden SL. Predicting outcome in patients with rhabdomyosarcoma: role of [(18)f] fluorodeoxyglucose positron emission tomography. Int J Radiat Oncol Biol Phys. 2014; 90:1136-1142.

19. Bisogno G, De Salvo GL, Bergeron C, et al. Vinorelbine and continuous low-dose cyclophosphamide as maintenance chemotherapy in patients with high-risk rhabdomyosarcoma (RMS 2005): a multicentre, open-label, randomised, phase 3 trial. Lancet Oncol 2019;20: 1566-75.

\section{LEGENDS}

TABLE 1. Demographic and clinical characteristics

TABLE 2. Site of relapses in the study cohort

TABLE 3. Prognostic factors A) Univariate analysis- EFS and OS B) Multivariate analysis- EFS and OS of whole cohort

Figure 1. Treatment schema used in the patients

Figure 2. Consort diagram of this retrospective study

Figure 3. A) EFS of whole cohort B) OS of whole cohort C) EFS based on PET response 3 months post definitive RT D) OS based on PET response 3 months post definitive RT

\section{Hosted file}

TABLE 1.pdf available at https://authorea.com/users/321709/articles/483545-prognosticsignificance-of-fdg-positron-emission-tomography-fdg-pet-response-post-chemotherapy-anddefinitive-radiotherapy-in-pediatric-rhabdomyosarcoma

\section{Hosted file}


TABLE 2.pdf available at https://authorea.com/users/321709/articles/483545-prognosticsignificance-of-fdg-positron-emission-tomography-fdg-pet-response-post-chemotherapy-anddefinitive-radiotherapy-in-pediatric-rhabdomyosarcoma

\section{Hosted file}

TABLE 3.pdf available at https://authorea.com/users/321709/articles/483545-prognosticsignificance-of-fdg-positron-emission-tomography-fdg-pet-response-post-chemotherapy-anddefinitive-radiotherapy-in-pediatric-rhabdomyosarcoma

\begin{tabular}{|c|c|c|c|c|c|c|c|c|c|c|c|}
\hline & $\begin{array}{l}\text { INDU } \\
\text { IEMO }\end{array}$ & $\begin{array}{l}\text { STION } \\
\text { HER }\end{array}$ & & & & $\mathrm{MA}$ & $\begin{array}{l}\text { AINTEI } \\
\text { EMOT }\end{array}$ & $\begin{array}{l}\text { NANC } \\
\text { HERA }\end{array}$ & & & \\
\hline VAC & VAC & VAC & VAC & VAC & VAC & VAC & VAC & VAC & VAC & VAC & VAC \\
\hline & & $R$ & & & & & OR & & & & \\
\hline VIE & VIE & VIE & VIE & $\mathrm{VIE}^{*}$ & $\mathrm{VIE}^{*}$ & VIE & VIE & VAC & VAC & VAC & VAC \\
\hline wo & w3 & W6 & w9 & W12 & W15 & W18 & W21 & W24 & W27 & W30 & W33 \\
\hline$\uparrow$ & & & & $\uparrow$ & & $\uparrow$ & & & & $\uparrow$ & \\
\hline FDG-PET CT & & & & LOCAL T & HERAPY & Y-RT & & $\begin{array}{l}\text { TU } \\
\text { FD }\end{array}$ & $\begin{array}{l}\text { MOR AS } \\
\text { G-PET }\end{array}$ & $\begin{array}{l}\text { SSESSI } \\
\text { CT }\end{array}$ & MENT \\
\hline
\end{tabular}

Figure 1. Treatment schema of the study. VAC: vincristine- iv push D1, D8, D15 (>

3years: $1.5 \mathrm{mg} / \mathrm{m}^{2}, 1-3$ years: $0.05 \mathrm{mg} / \mathrm{kg}$, <1year: $0.025 \mathrm{mg} / \mathrm{kg}$ ), actinomycin-D- iv push D1 (>3years/1-3years: 0.045mg/kg, <1year: 0.025mg/kg), cyclophosphamideiv over 30 minutes D1 (>3years: 2200mg/m2, 1-3years: 73mg/kg, <1year: 36mg/kg) with iv mesna $500 \mathrm{mg} / \mathrm{m}^{2}$ at $0,3,6,9$ hours of cyclophosphamide D1, VIE: vincristine$1.5 \mathrm{mg} / \mathrm{m}^{2}$ iv push D1, D8, D15, ifosfamide- $1800 \mathrm{mg} / \mathrm{m}^{2}$ iv infusion over $2 \mathrm{hrs}$ with iv mesna $600 \mathrm{mg} / \mathrm{m}^{2}$ at $0,3,6,9$ hours of ifosfamide D1-D5, etoposide- $100 \mathrm{mg} / \mathrm{m}^{2}$ iv over 1-2 hours D1-D5, RT- Radiotherapy, FDG PET- Fluorodeoxy-Positron Emission Tomography. * Dactinomycin is omitted during concurrent RT to head, neck and truncal sites, and etoposide is omitted during concurrent RT to any site 


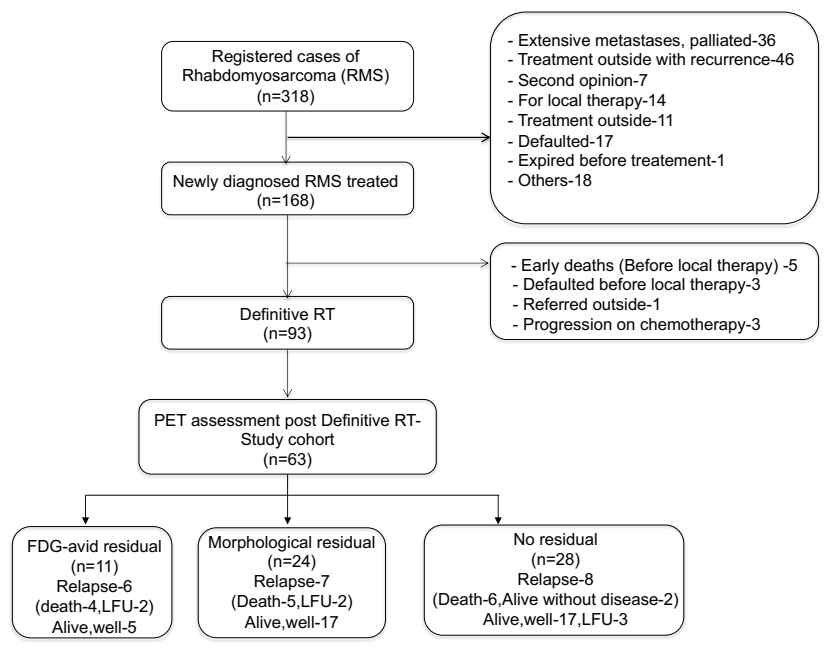

Figure 2. Consort diagram of this study 
A)

Event Free survival
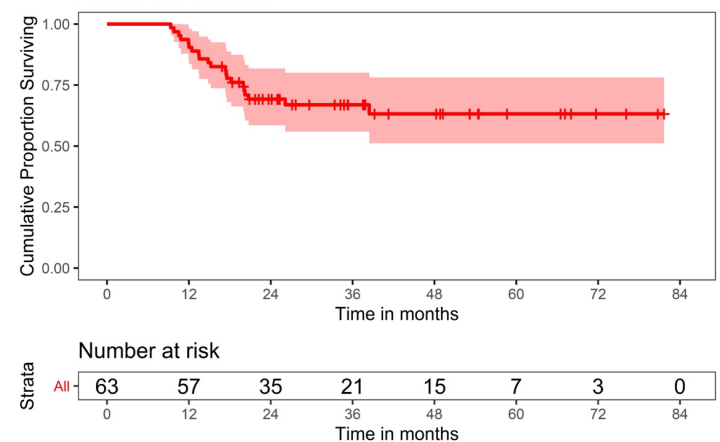

B)

Overall survival

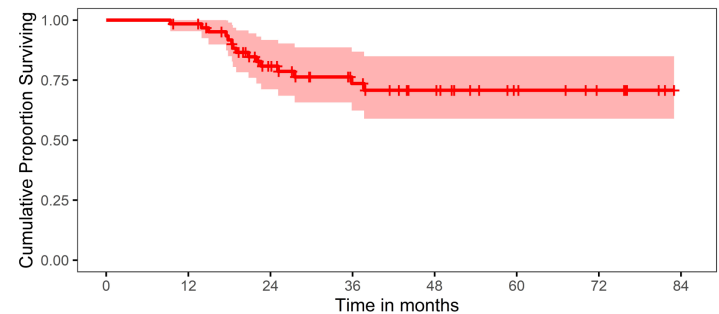

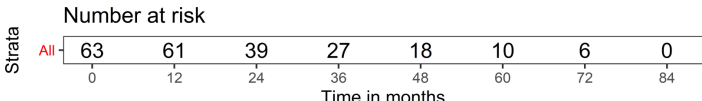

